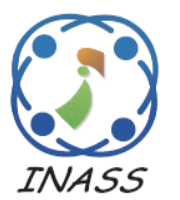

\title{
Time-Frequency Representations Adapted to the Characterization of Steels Damaged by the Environment
}

\author{
Lahcen Mountassir $^{1 *}$, Touriya Bassidi ${ }^{1}$, Salma Aziam $^{1}$, Hassan Nounah $^{1}$ \\ ${ }^{1}$ Laboratory of Metrology and Information Processing, Faculty of Science, Ibn Zohr University, Agadir, Morocco \\ *Corresponding author's Email: mountassir.lahcen8@gmail.com
}

\begin{abstract}
This article intends to present a time-frequency representations adapted to the characterization of steels damaged by the environment. These techniques are applied for analyzing experimental acoustic signal backscattered by corroded steel immersed in water. These signals are processed using a spectrogram analysis, Pseudo Wigner-Ville Distribution PWV and continuous wavelet, whose interest is to provide a signal representation in time-frequency. The goal here is whether each pattern of representation is due to corrosion or default in the plate. The use of timefrequency representations for characterizing steels damaged by the environment proves interesting because of their ability to localize the energy of the signals in a two-dimensional plane. However, bearing in mind that these representations should check drastic properties about the studied signal, choose a representation adapted case by case. The results obtained highlight the advantage of using time-frequency representations for the study of the acoustic wave's propagation in corroded steels.
\end{abstract}

Keywords: Steel, Corrosion, HCl, Transmission, Reflection, Time-frequency, Ultrasound, Nondestructive testing.

\section{Introduction}

The non-destructive testing operation of an object does not limit to detecting potential defects [1-3]. Even if the choice of process, methodology, and hardware was carried out beforehand, consider a procedure with the following objectives: reliability of the examination, reproducibility, identification, characterization and finally archiving of control results [1]. In the industry, we are often forced to put many pieces of controls for reasons of security and cost [4]. The presence of a crack or corrosion is too small; it can present a permanent risk that can cause severe bodily and material damage [4-5]. The main advantage of using ultrasonic non-destructive testing is to restore the controlled piece as it was before the control [6].

Among the many methods, we choose those that retain critical notions of time and frequency corresponding to a distribution of the signal energy in the time-frequency plane $[7,8]$. Thus, it becomes possible to follow the evolution of the main spectral components of the signal versus time and vice versa. Amongst all the techniques of the Cohen class [9$10]$, the class that includes all the non-parametric time-frequency methods based on Fourier transform [11], that are the spectrogram, Pseudo Wigner-Ville, and wavelet transforms technical's, are retained in this article for their unusual properties $[7,8]$.

Our primary objective of this study is to characterize the corroded steel plate by using timefrequency representation. In other terms, our goal in this work is the characterization of the phenomena involved in the ultrasonic propagation used the processing tools of the time-frequency representations. The advantage of this method, it enables efficient diagnosis of the signal which it deploys energy in the time-frequency plane, this method able to follow the changes that occur in the signal (compressions / dilations, translations in both time and frequency domains), these allow representation us to account the attenuation and dispersion caused by the propagation in the material. 


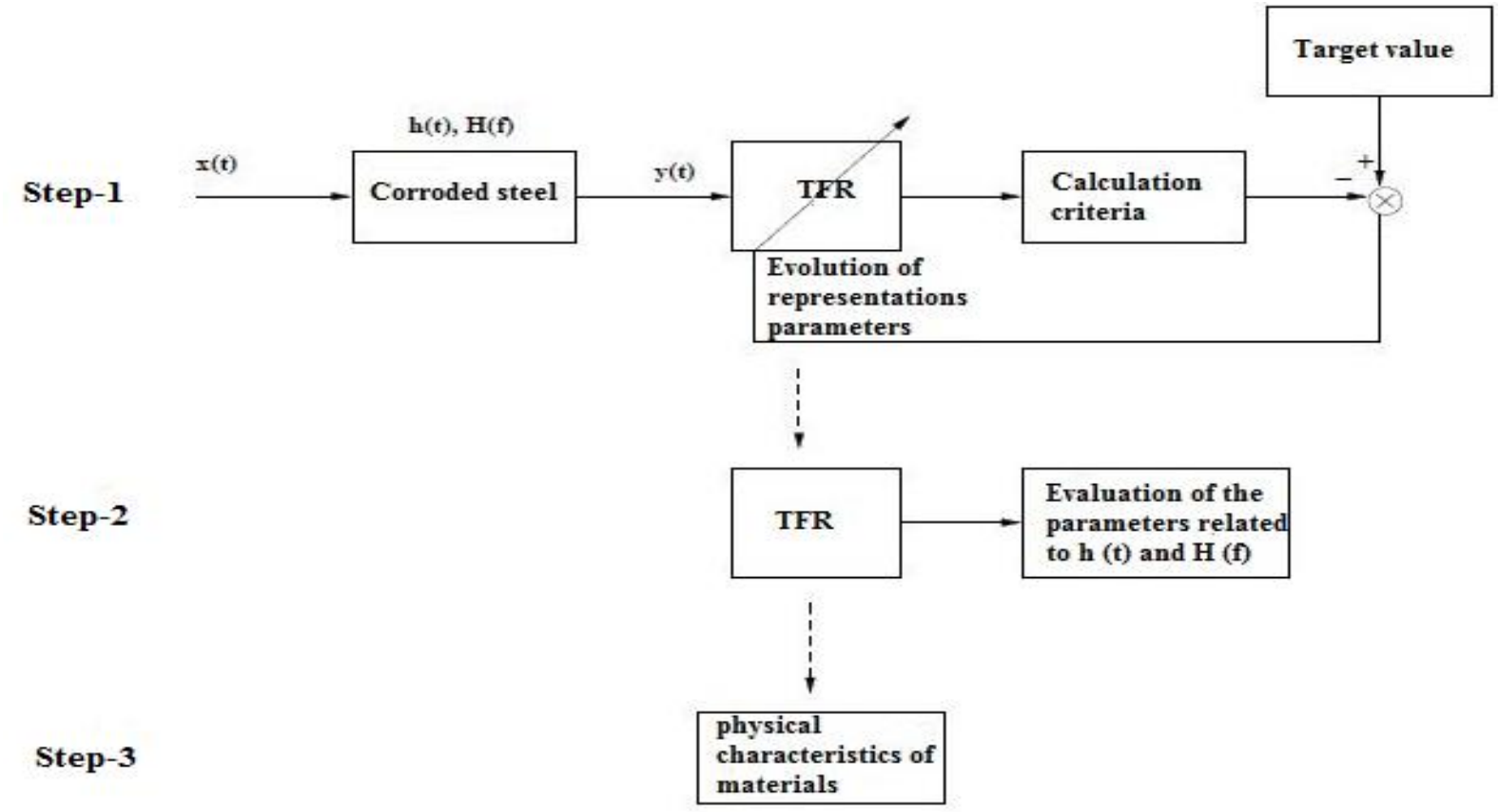

Figure.1 The principle for the characterization of steel corroded with time-frequency representations (TFR). Step 1: several time-frequency representations are used to illustrate the properties of the signal coming the material. Each performance is evaluated by calculating a criterion. Step 2: the corresponding time-frequency representation at best test is maintained. Designed to estimate the parameters of the impulse response of the medium. Step 3: in finally, we go to the physical characteristics of the materials from the parameters identified in step 2.

The results showed that the TFR have superior performance, regarding resolution and an excellent legibility as compared to another signal processing method.

The paper is organized as follows: Section 2 provides an overview of the ultrasound method. Section 3 describes the time-frequency technique proposed in this study. Section 4 presents the experimental results and discussion.

\section{Experimental Study}

The ultrasonic testing is to corrosion a nondestructive inspection method for detecting, locating, evaluating and monitoring the degradation of metals [12, 13]. The consequences of corrosion are well known, and technical implementations are expensive to repair [14-15]. The application of an ultrasound control can detect damage or protective coatings such as blistering, the exposed metal or metal itself $[12,13,16]$.

\subsection{Sample preparation}

Before the ultrasonic characterization of the samples, you must first prepare the form of plates. The experimental device used for this work requires the samples must meet certain dimensions in thickness, width, and length. The Table 1 shows the samples of steel plates studied in this work and the immersion time in the acid solution $(\mathrm{HCl})$ used to initiate corrosion in some plates among the samples to be tested.

\subsection{The ultrasonic technique by transmission}

Figure 2 show the experimental setup of the transmission method. The experimental design for this study is composed primarily of two same ultrasonic transducers plans for center frequency $5 \mathrm{MHz}$ for each one. The first transducer is a transmitter excited in pulsed mode with a pulse generator Sofranel Model 5052PR. Sensor response following to this excitation is reflected by the generation of ultrasonic waves which propagate in the first coupling medium (in our case: water) then they cross the steel specimen. They will be captured after they pass through a second coupling medium (here: water) by the second transducer that plays a role of the receiver. The latter returns the ultrasound signal picked up towards the generator to amplify, and then the amplified ultrasonic signal will be sent to the oscilloscope HP54600B to visualize it. After averaging operation to improve the signal/noise ratio signal visualized, it will be forwarded to the computer through the port via GPIB IEEE National data acquisition card Instrument controlled by the LabView software. We have developed a platform with Labview which allows us to easily manipulate 
Table 1. Presentation of steel samples to characterize.

\begin{tabular}{|c|c|c|c|c|c|}
\hline The plate & $\begin{array}{c}\text { Thickness } \\
(\mathrm{mm})\end{array}$ & Width $(\mathrm{cm})$ & $\begin{array}{c}\text { Length } \\
(\mathrm{cm})\end{array}$ & $\begin{array}{c}\text { The density } \\
\left(\mathrm{Kg} / \mathrm{m}^{3}\right)\end{array}$ & $\begin{array}{c}\text { Immersion time in } \\
\mathrm{HCl}\end{array}$ \\
\hline Steel 1 & 2 & 7,71 & 10,01 & 7471,12 & $2 \mathrm{~h}$ in $\mathrm{HCl}(5 \mathrm{~N})$ \\
\hline Steel 2 & 3 & 9,91 & 10,33 & 7268,38 & $2 \mathrm{~h} \mathrm{in} \mathrm{HCl}(5 \mathrm{~N})$ \\
\hline Steel 3 & 3 & 7,62 & 8,78 & 7059,9 & $4 \mathrm{~h}$ in $\mathrm{HCl}(5 \mathrm{~N})$ \\
\hline Steel 4 & 2 & 7,96 & 7,98 & 7419,6 & $24 \mathrm{~h}$ in $\mathrm{HCl}(5 \mathrm{~N})$ \\
\hline oxidized Steel & 3 & 7,56 & 9,01 & 7083,056 & - \\
\hline
\end{tabular}

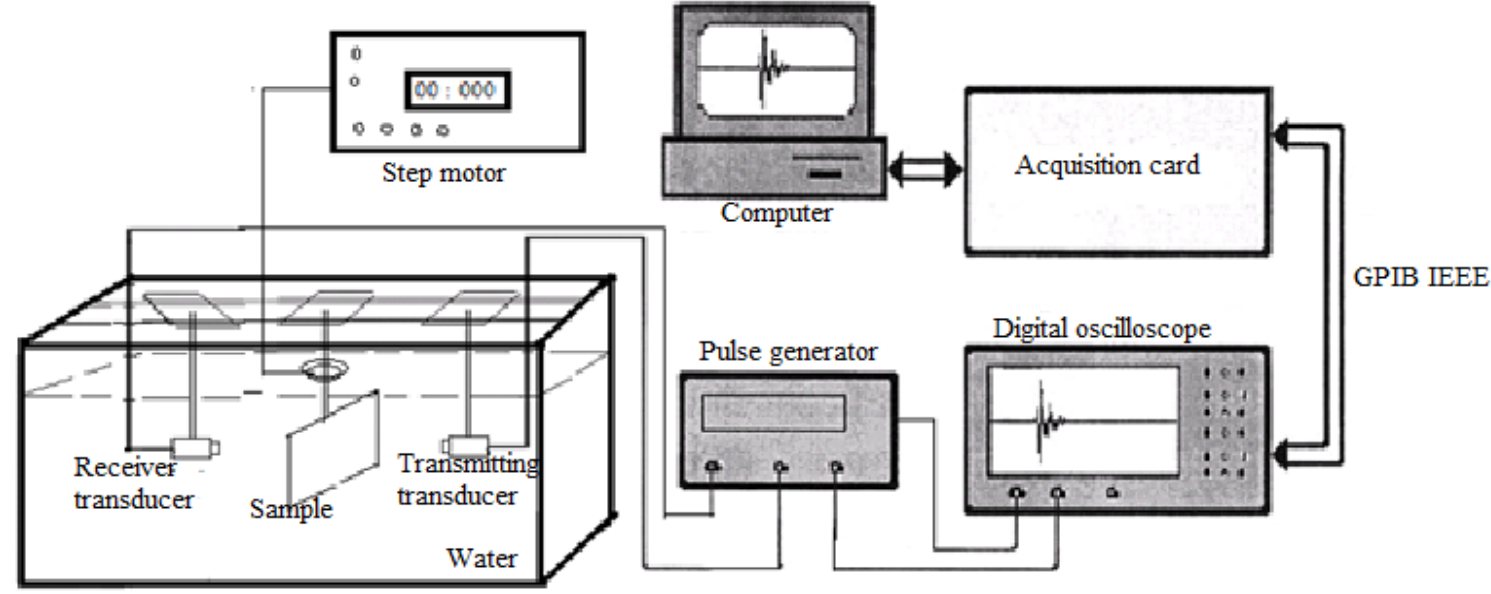

Figure. 2 Schematic showing the experimental setup of transmission method

the signals acquired their registration and calculation of the FFT automatically for each signal.

\subsection{The ultrasonic technique by reflection}

Figure 3 show the experimental setup of the reflection method. In this approach, the transducer used $5 \mathrm{MHz}$ for center frequency, operates alternately as a transmitter and receiver. The pulse generator electrically excites the transducer. The transducer senses the backscattered acoustic pressure. The received signal moves through the same cable as the transmission signal and enters the connector $\mathrm{T} / \mathrm{R}$ from which the transmission signal has been sent. Through a gate and an incorporated delay system into the pulse generator, the received signal is visualized by connecting a coaxial cable the output connector to a channel of the oscilloscope HP54600B control, synchronized with the generator pulse. The oscilloscope is related to the computer via an IEEE bus for signal processing.

\section{Time-frequency representation (TFR) of the acoustic response}

The majority of the time-frequency methods are represented by the Cohen class $[9,10]$. From among the many performances of this class time-frequency, we selected the Pseudo Wigner-Ville distribution (PWV), the spectrogram and the wavelet transform, for their valuable properties.

\subsection{Distribution Pseudo Wigner-Ville PWV}

Generally, for the convenience of calculation in the presence of a signal at infinite temporal support (colossal in practice), it performs a smoothing operation frequency of the PWV through a window 

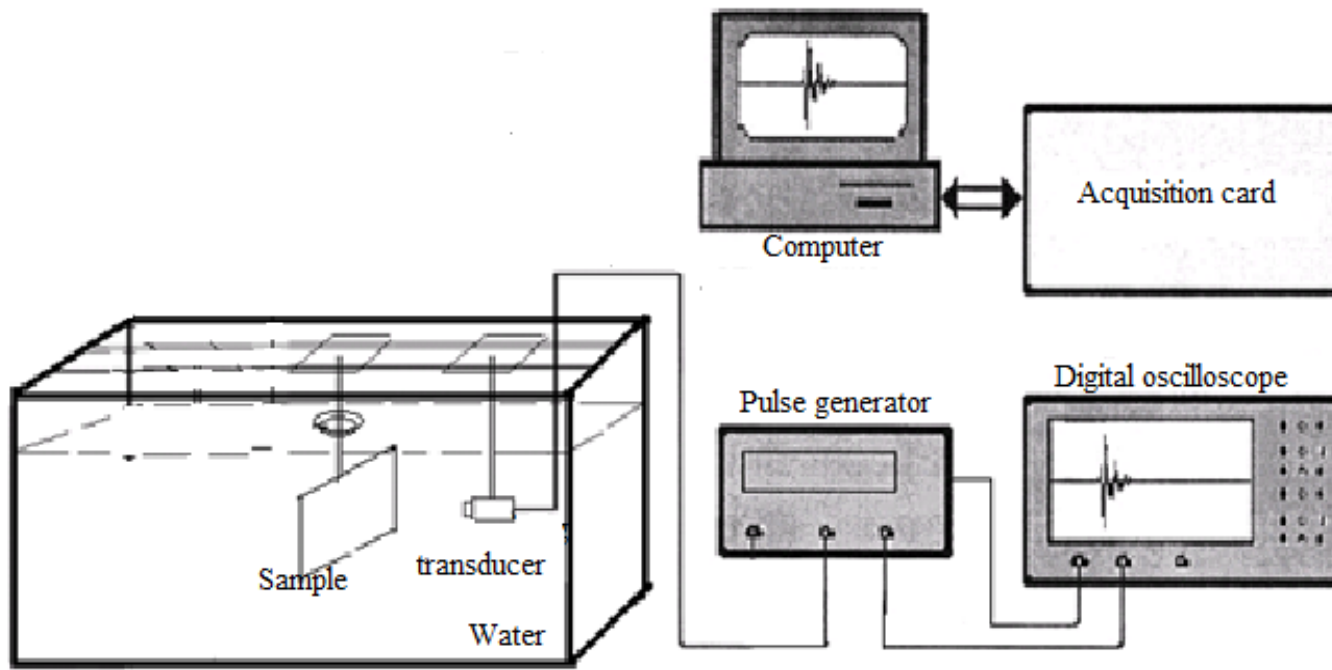

GPIB IEEE

Figure. 3 Schematic showing the mounting of the reflection technical

$h_{T}(\mathrm{t})$. The PWV using two smoothing window $\mathrm{h}(\mathrm{t})$ and $\mathrm{g}(\mathrm{t})$. These smoothing windows are introduced into the Wigner-Ville distribution definition to allow good control, either in time $(\mathrm{g})$ or in the frequency (h) $[7,9,17]$. The expression of this representation is defined by:

$$
P W V_{y}(t, v)=\int_{R} h_{T_{0}}(\tau) y\left(t+\frac{\tau}{2}\right) y^{*}\left(t-\frac{\tau}{2}\right) e^{-2 j v \tau \pi} d \tau
$$

With $h_{T_{0}}(t)$ : a window being written in the form

$$
h_{T_{0}}(\tau)=\mid\left(\left.h\left(\frac{\tau}{2}\right)\right|^{2} g(t-u)\right.
$$

Where $\mathrm{h}(\mathrm{t})$ is a frequency smoothing window and $\mathrm{g}(\mathrm{t})$ is a temporal smoothing window and $\mathrm{y} *(\mathrm{t})$ is the complex conjugate of the analytical signal $\mathrm{y}(\mathrm{t})$.

\subsection{Distribution spectrogram}

The Fourier transform short term (STFT) can be interpreted as the weighted Fourier analysis of successive portions, a time window (Gabor, hamming, ...) [11], this principle is equivalent to the approximation of the signal by a set of elementary functions semi-localized simultaneously in time and frequency $[7,17]$ :

$$
\operatorname{STFT}_{x_{a}}(t, u)=\int_{-\infty}^{+\infty} x_{a}(\tau) h_{t, u}^{*}(\tau) d \tau=\int_{-\infty}^{+\infty} x_{a}(\tau) h(\tau-t) e^{-2 \pi j u \tau} d \tau
$$

In practice, using the spectrogram, which is the modulus, squared of the STFT. When the values of STFT are complex, the square module ensures that the spectrogram value will always be a real value.
The spectrogram (SP) is then defined as an energy density. The energy density function or spectrogram of the signal corresponding to the window $h$ is given by:

$$
S P_{x_{a}}(t, u)=\left|S T F T_{x_{a}}(t, u)\right|^{2}
$$

The spectrogram can be seen as a smoothed version of the Wigner-Ville distribution of the signal $x_{a}(t)$ is shown as follows:

$$
S P_{x}(t, u)=\iint W_{x}(\tau, \mu) W_{h}(\tau-t, \mu-u) \frac{d \tau d \mu}{2 \pi}
$$

Where $W_{x}$ and $W_{h}$ are the Wigner-Ville distribution of the signal $x(t)$ and the window $h$ respectively.

\subsection{Wavelet transform}

The wavelet $\psi$ is a function used in the time scale transformations, where a comparison of the signal is made of a collection of elements where the number of oscillations remains constant when the time support (time where mother wavelet is defined) decreases. This function is dilated with a scale parameter $a$ and translated in time by $u$. The wavelet transform is, too, based on the projection method of a signal on the basis of functions, which are in this case wavelets. The definition of a wavelet is given by $[7,20,21]$ :

$$
\psi_{u, a}(t)=\frac{1}{\sqrt{a}} \psi\left(\frac{t-u}{a}\right) \quad a, u \in R, a>0
$$


$a$ is called the scale factor and $u$ the temporal translation factor. The wavelet transform is defined by:

$\operatorname{TOx}(u, a)=\int_{-\infty}^{+\infty} x(t) \frac{1}{\sqrt{a}} \psi^{*}\left(\frac{t-u}{a}\right) d t$

As part of the continuous wavelet transform, $a$ and $u$ varies continuously [22].

\subsection{Application example of TFR}

The figure 4 and 5 shows an exemplary corrosion detection hidden in Aluminium using wavelet transform.

\section{Results and Discussion}

The method used is based on a representation of the experimental signal in the time-frequency plane. The expected properties of an RTF applied to a signal coming from a material must make it possible to monitor its evolution in the time-frequency plane with a maximum resolution along its propagation.

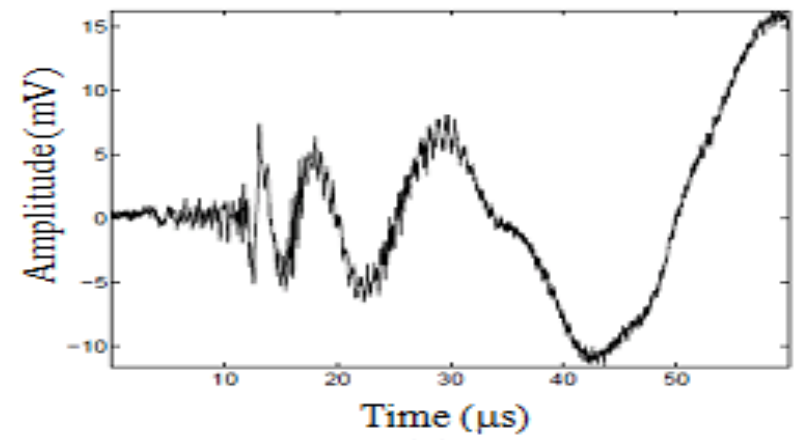

(a)

\subsection{Reflexion by focusing transducer of centre frequency $20 \mathrm{MHz}$}

\subsubsection{Result by time frequency representation}

The figure 6-8 represents the time signals (Figures 6-a, 7-a, 8-a) and the time-frequency representations (Figures 6-b, 7-b, 8-b) corresponding to a steel plate in the good area, a plate area corroded during $7 \mathrm{~h}$ and the other area destroyed during 26h. We note that the general aspect of the temporal signals is difficult to compare, but it is possible to see, between $2 \mu$ s and $7 \mu$ s, the amplitude decreases with increasing immersion time, we can say that there is a smoothing of our signal for corrosion presence. On the scalogram, the most attractive region is that of high frequencies, because the modes are partially disappearing in the figure (7b), and then completely in figure (8-b). This area seems within the rectangle from $5 \mu$ s to $10 \mu$ s for high frequencies. We can assume that the presence of corrosion is characterized by the disappearance or attenuation of some modes.

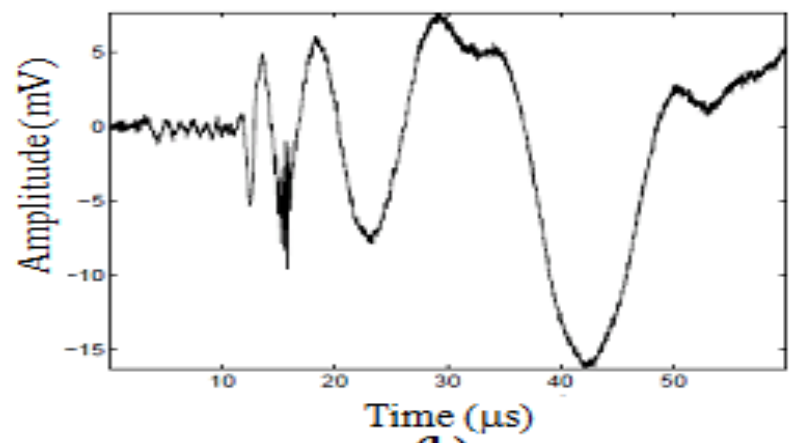

(b)

Figure. 4 Time signals corresponding, healthy area (a) and corroded area (b) [22].

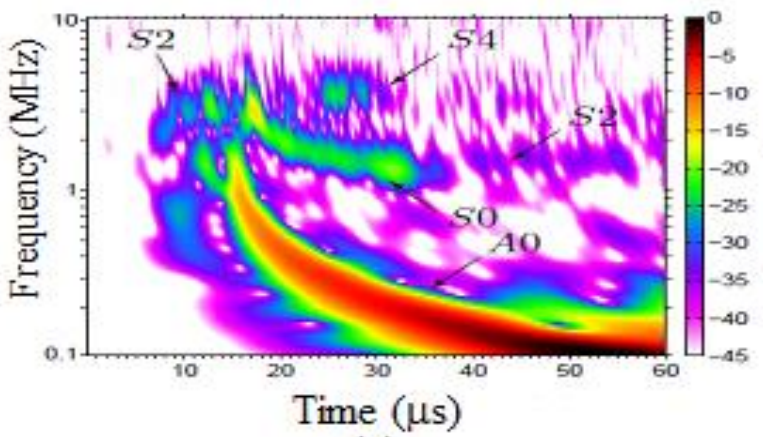

(a)

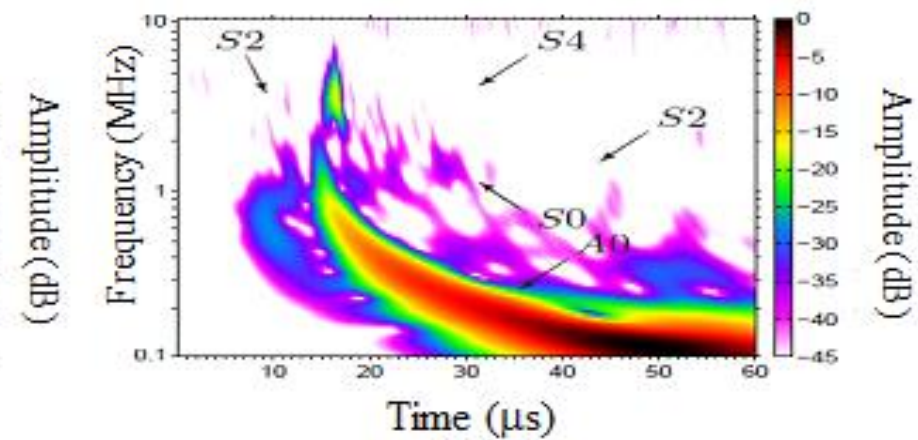

(b)

Figure. 5 Scalograms a signal in healthy area (a) and corroded area (b), the presence of corrosion causes attenuation modes $S 0, S 2$ and $S 4$ [22]. 


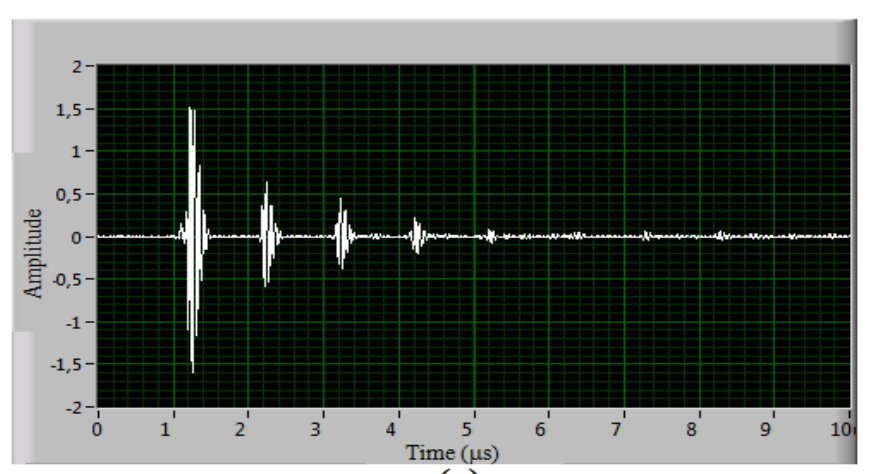

(a)

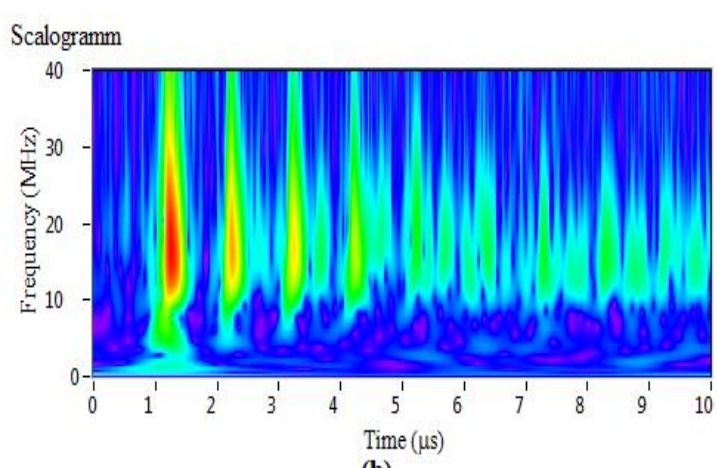

(b)

Figure. 6 Time signal in healthy area (a), the scalogram of time signal (b).



(a)



(b)

Figure. 7 Time signal in corroded area $7 \mathrm{~h}(\mathrm{a})$, the scalogram of time signal (b).

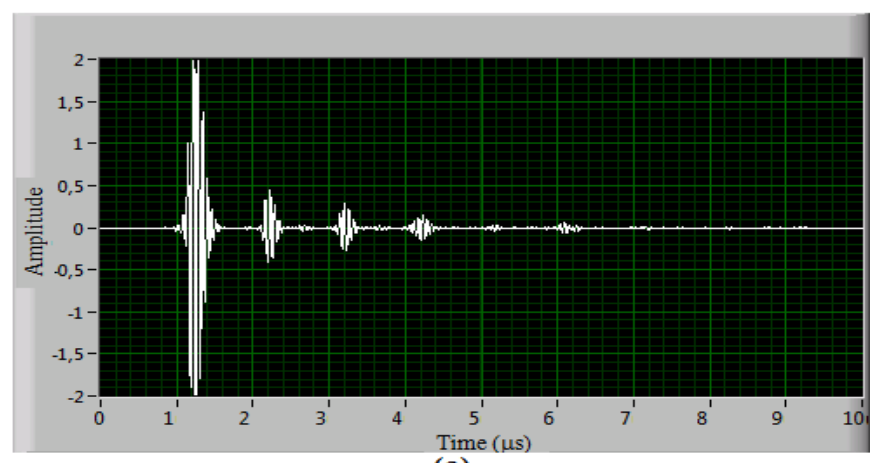

(a)

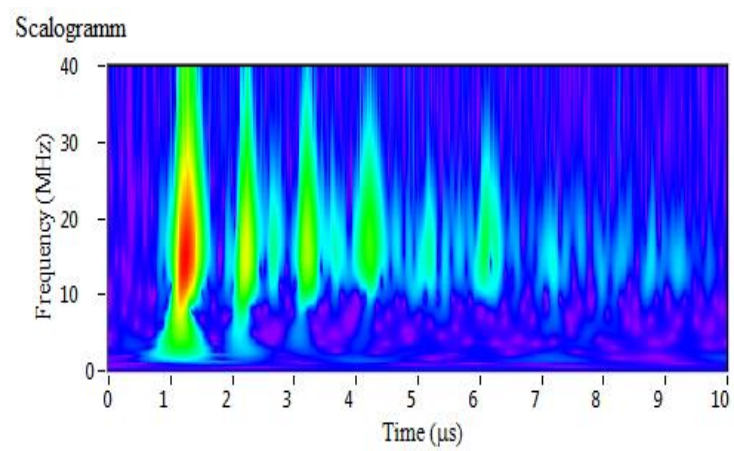

(b)

Figure. 8 Time signal in corroded area $26 \mathrm{~h}(\mathrm{a})$, the scalogram of time signal (b).

\subsection{Transmission oblique incidence}

\subsubsection{Result by time frequency representation}

The figure 9 show the time signal and PWV representations corresponding to two steel plates corroded: the first (Figures 9-a, 9-c) is immersed in hydrochloric acid for $2 \mathrm{~h}$ and the second (Figures 9-b, 9-d) is immersed in hydrochloric acid for $4 \mathrm{~h}$. We notice a difference between the two configurations in the number of modes that propagate in the two plates; in fact, the number of modes in the steel 3 is reduced compared to that of steel 2 .

The figure 10 show PWV representations corresponding to two steel plates corroded: the first (Figures 10-a, 10-c) is oxidized and the second (figures 10-b, 10-d) is immersed in hydrochloric acid for $2 \mathrm{~h}$. We notice a difference in the two configurations at the clarity of the modes propagating in the two plates: indeed, the modes in the oxidized plate are bright compared to those of the steel 2 plate. The shape of modes is complete in the oxidized plate, unlike the steel 2 plate whose shape of the last mode is attenuated. 


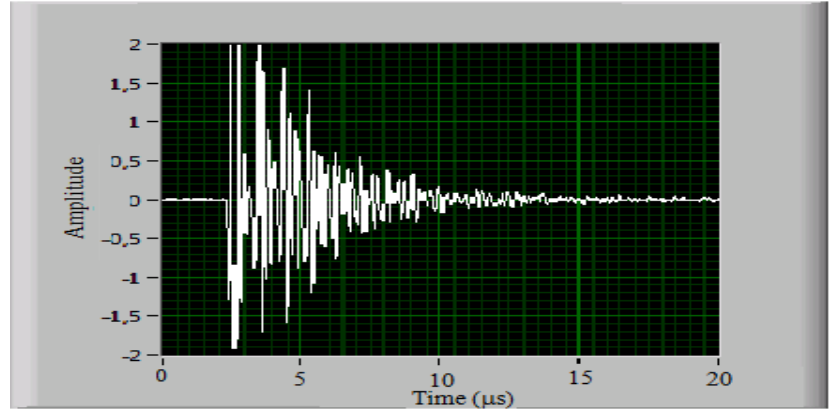

(a)

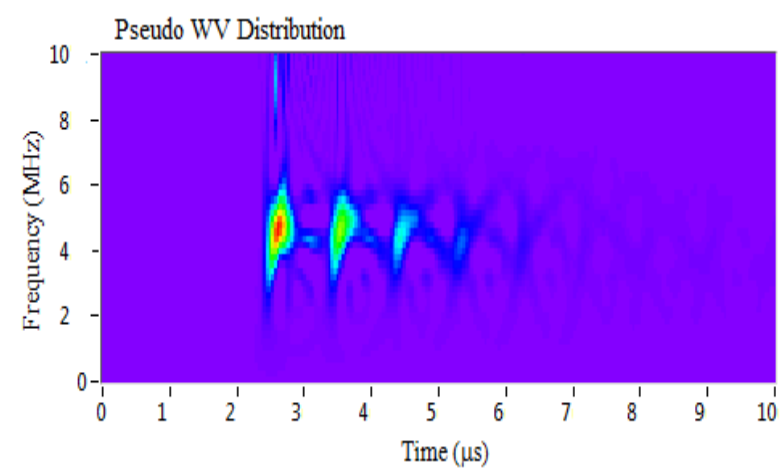

(c)

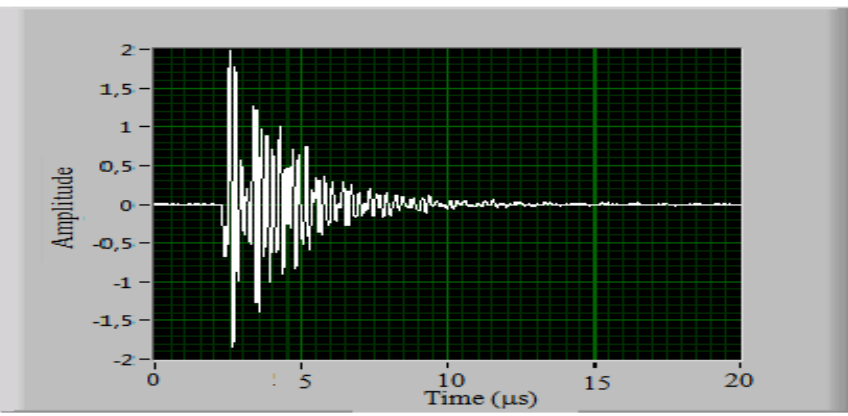

(b)

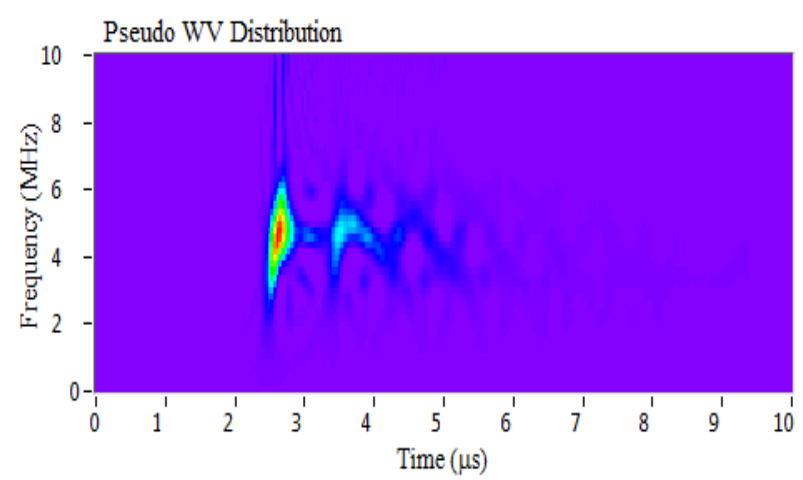

(d)

Figure. 9 (a, b) Time signal of steel 2 and steel 3, (c, d) PWV of temporal signal steels 2 et 3.

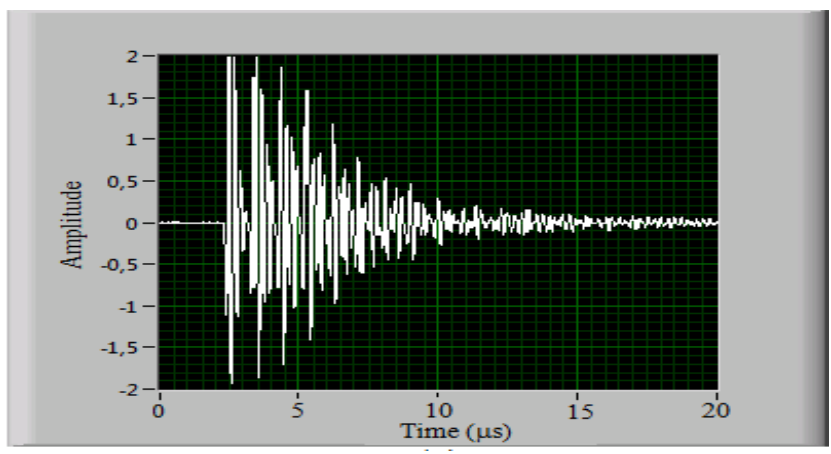

(a)

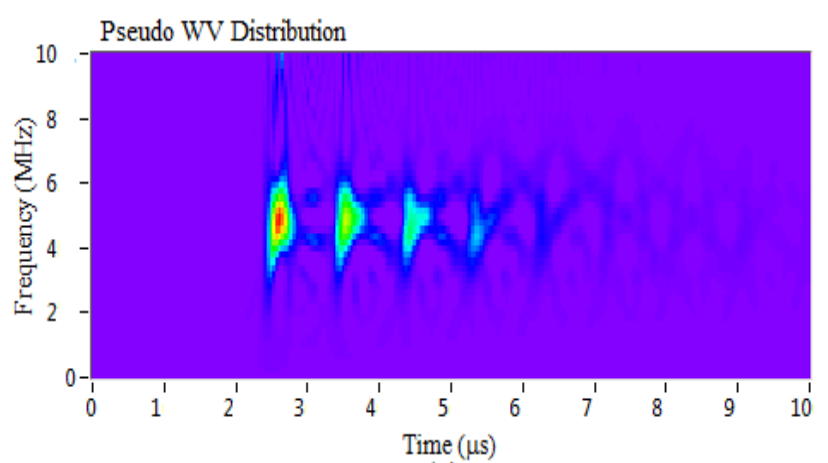

(c)

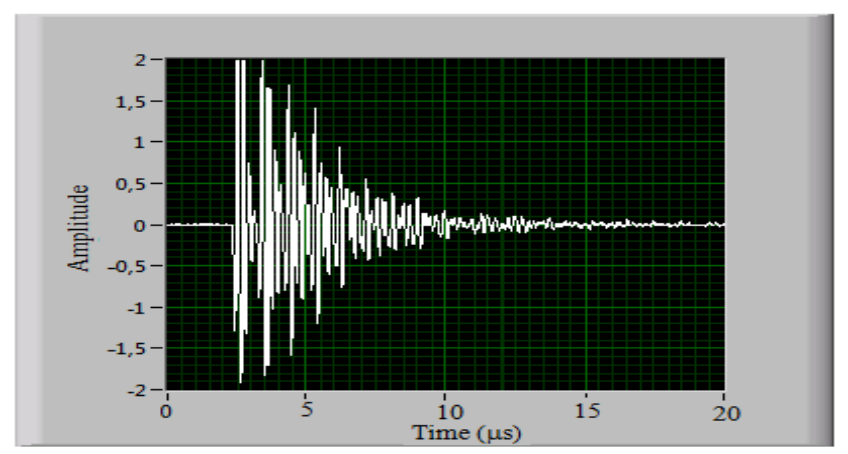

(b)

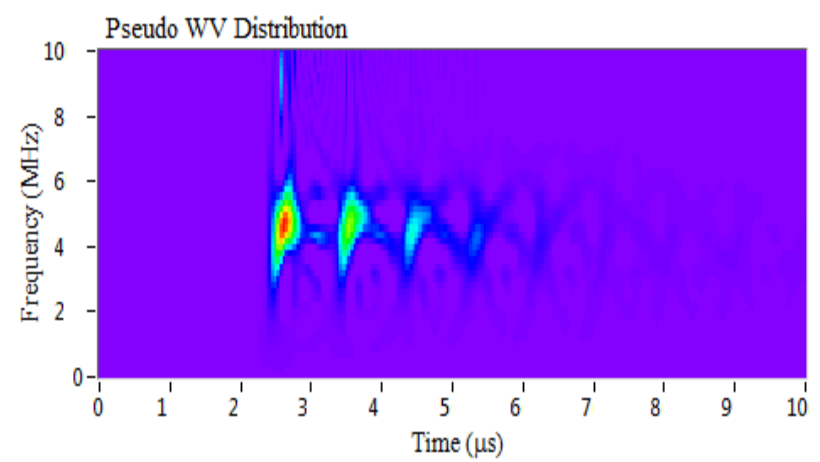

(d)

Figure. 10 (a, b) Time signal of oxidized steel and steel 2, (c, d) PWV temporal signal of oxidized steel and steel 2. 


\subsection{Steel immersion in $\mathrm{HCl}(1.5 \mathrm{~N})$}

Figure 11 represent the temporal signals (Figures 11-a,11-b), spectrograms (Figures 11-c,11-d) and scalograms (Figure 11-e,11-f) corresponding to two steel plates corroded: the first is that we have studied and the second is immersed in $\mathrm{HCl}(1.5 \mathrm{~N})$ during 26h. We notice a difference in the spectrograms at the shape, clarity, and location of the modes that propagate in the two plates: indeed the modes in the original plate are bright compared to those of the new plate. Also, the form of the modes is complete in the old plate instead of the new plate whose shape of modes is varied and

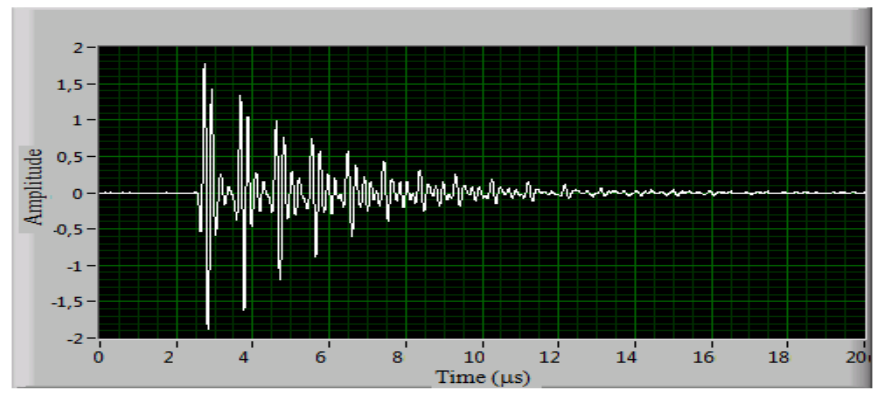

(a)

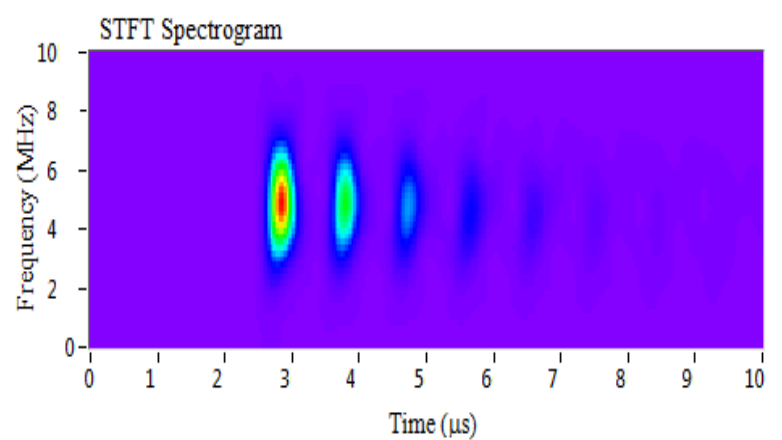

(c)

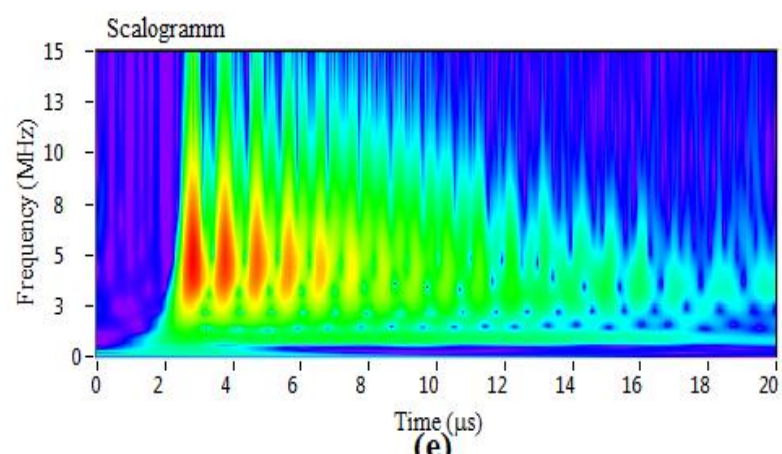

(e) attenuated. The location of the modes that are shifted by $1 \mu$ s compared to the old one. Concerning scalograms, we observe that the shape of the patterns located between $2 \mu \mathrm{s}$ and $8 \mu \mathrm{s}$ in the new plate is varied, and between $10 \mu \mathrm{s}$ and $20 \mu \mathrm{s}$, the modes are attenuated.

Figure 12 represent the temporal signals (Figures 12-a,12-b), PWV distribution (Figures 12-c,12-d) and scalograms (Figures 12-e,12-f) corresponding to two steel plates corroded: the first is that we have studied and the second is immersed in $\mathrm{HCl}(1.5 \mathrm{~N})$ during 26h. We notice a difference in the PWV distribution at the shape clarity, and location of Lamb modes that propagate in the two plates:

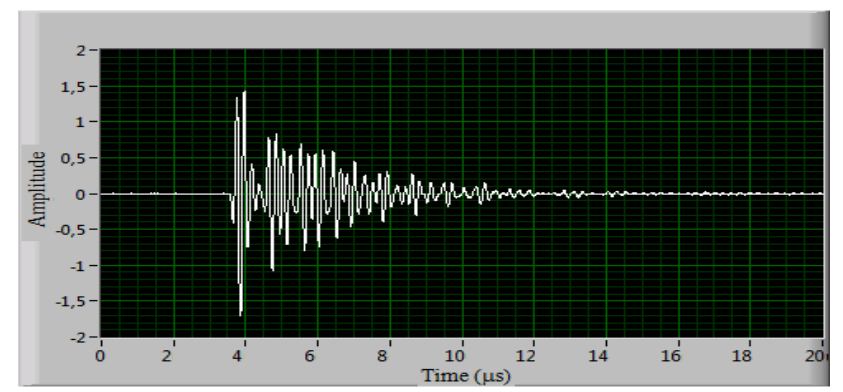

(b)

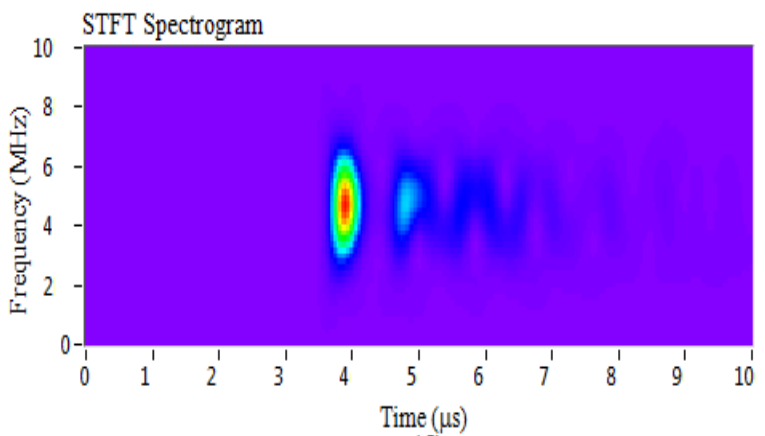

(d)

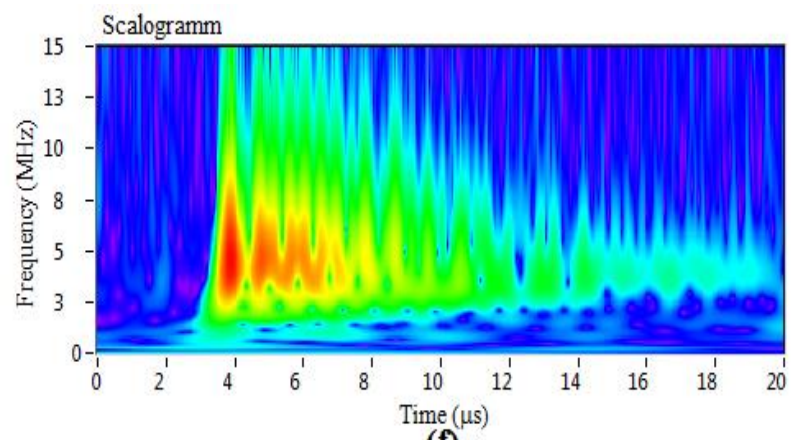

(f)

Figure. $11(\mathrm{a}, \mathrm{b})$ Time signal of steel 2 and steel $2(+26 \mathrm{~h}$ in $\mathrm{HCl} 1,5 \mathrm{~N}),(\mathrm{c}, \mathrm{d})$ spectrograms of steel 2 and steel 2 $(+26 \mathrm{~h}$ in $\mathrm{HCl} 1,5 \mathrm{~N}),(\mathrm{e}, \mathrm{f})$ scalograms of steel 2 and steel $2(+26 \mathrm{~h}$ in $\mathrm{HCl} 1,5 \mathrm{~N})$. 


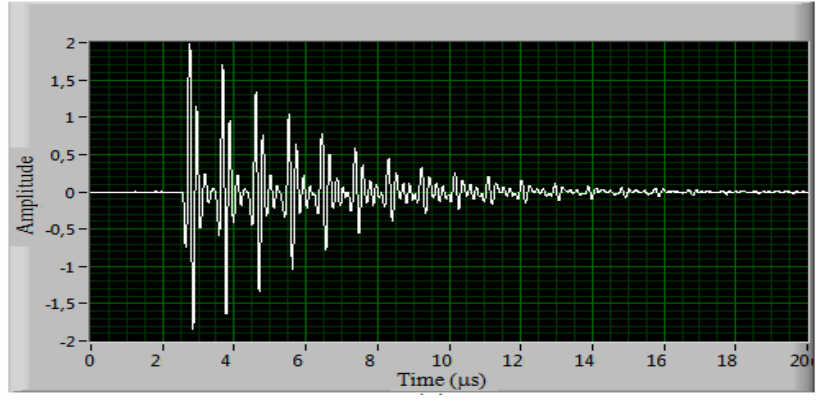

(a)

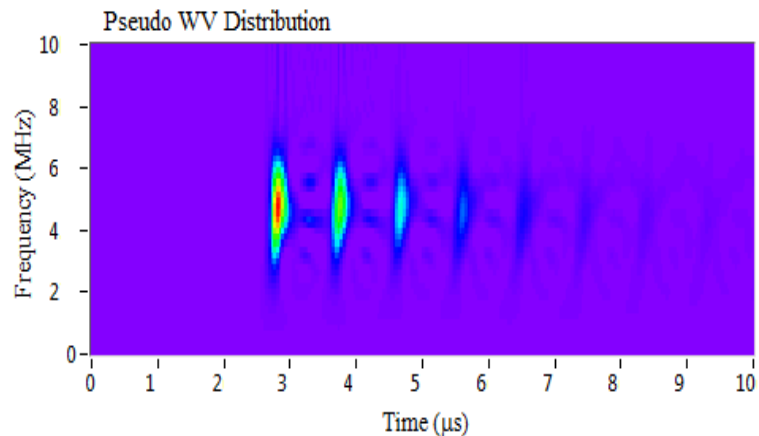

(c)

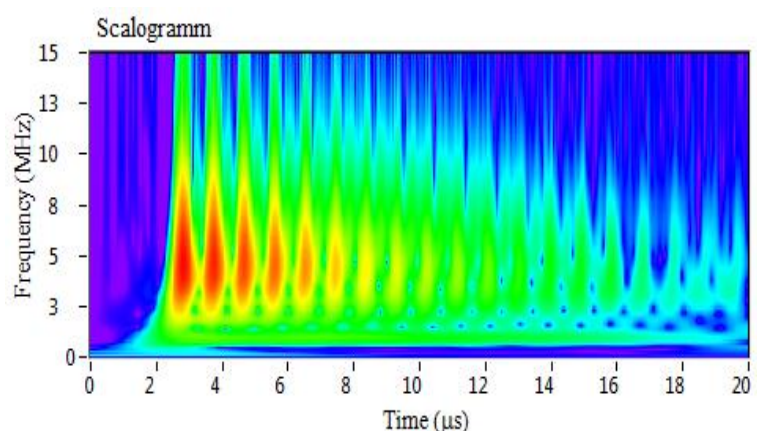

(e)



(b)



(d)

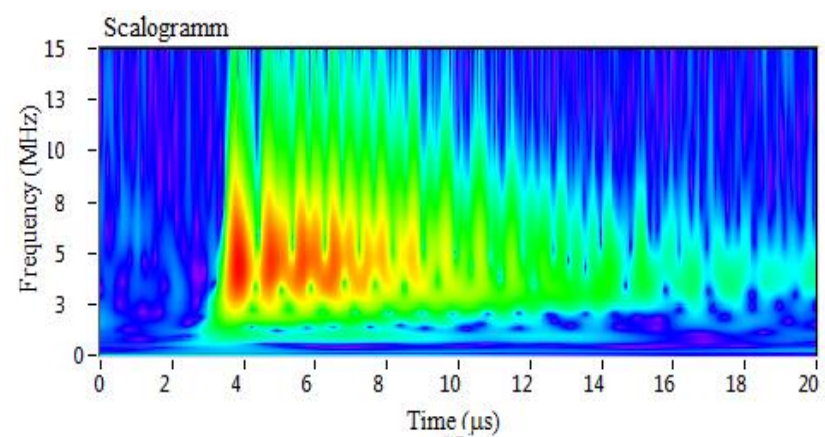

(f)

Figure. 12 (a, b) Time signal of steel 3 and steel $3(+26 \mathrm{~h}$ in $\mathrm{HCl} 1,5 \mathrm{~N}),(\mathrm{c}, \mathrm{d}) \mathrm{PWV}$ distribution of steel 3 and steel 3 $(+26 \mathrm{~h}$ in $\mathrm{HCl} 1,5 \mathrm{~N}),(\mathrm{e}, \mathrm{f})$ scalograms of steel 3 and steel $3(+26 \mathrm{~h}$ in $\mathrm{HCl} 1,5 \mathrm{~N})$.

indeed the modes in the original plate are bright compared to those of the new plate. Also, the form of the modes is complete in the old plate instead of the new plate whose shape of modes is varied and attenuated. The location of the modes that are shifted by $1 \mu$ s compared to the old one. Concerning scalograms, we observe that the shape of the patterns located between $2 \mu \mathrm{s}$ and $8 \mu \mathrm{s}$ in the new plate is varied, and between $10 \mu$ s and $20 \mu \mathrm{s}$, the modes are attenuated.

The TFR method we achieved the existence of a relationship between the immersion duration of steel plate in the acid solution and attenuation modes in the steel plates corroded. This significant result is that the fact of modes attenuation following increasing immersion time in hydrochloric acid. This result clearly shows the ability of this technique to detect the effect of corrosion on the corroded steel plates in a reliable and non-destructive way.

The time-frequency analysis of the Wigner-Ville, spectrogram, and wavelets, acoustic from signals backscattered by an elastic plate, made it possible to analyze and identify the waves propagating in this dish. This analysis also allows us to follow, the frequency evolution of each wave in the timefrequency plane generated by these representations. Finally, from this joint time-frequency analysis of the ultrasonic signal, several characteristics associated with acoustic propagation can be demonstrated. These are dispersion and attenuation of waves as a function of time and resonance frequencies. This type of analysis can, therefore, be used as a means to understand the mechanism of ultrasonic propagation and to suggest a better interpretation of the phenomenon. 


\section{Conclusion}

The characterization of the steel plates is the object of a vast number of experimental and theoretical studies. In this study, we used a new signal treatment approach based on the timefrequency representation for characterizing corroded steel plates. The proposed method relies on the presentation of time signals backscattered by the corroded steel plates in the time-frequency plane. In the current research, we tested the ability of timefrequency representations to follow the changes that occur in the signal steels plate of different immersion duration in the acid solution. Due to its high time-frequency resolution and cross-checking, the reassigned time-frequency representations should be active in analyzing the acoustic signals. Time-frequency representations give access to different types of qualitative and quantitative information. Among the qualitative information, we have shown that it makes possible to follow the evolution of the frequency content of the waves as a function of time; and among the quantitative, this technique can give the attenuation and dispersion of the ultrasonic waves. The method employed shows an almost perfect agreement between this work and literature. This approach can be generalized to other damaged materials. This study reveals the advantages of the implementation of the method proposed in the ultrasonic testing. This research can be used as a new method for the characterization of corroded steel.

The RTF are appropriate tools for the study of the corroded steel acoustic properties. These can measure the frequency dependency of the corrosion and consider a solution to the inverse problem of finding the physical parameters of steel from the experimental signal. In the further researches, we will concentrate on the beginning of corrosion and the detection of the corroded layer thickness by exploiting the time-frequency representations.

\section{References}

[1] G. Sposito, C. Ward, P. Cawley, P. B. Nagy \& C. Scruby, "A review of non-destructive techniques for the detection of creep damage in power plant steels." Ndt \& E International, Vo. 43, No. 7, pp. 555-567, 2010.

[2] CU. Grosse, M. Ohtsu, eds. "Acoustic emission testing". Springer Science \& Business Media, 2008.

[3] A. Lambert, "Non-destructive ultrasonic testing." Heat treatment, Vol. 230, pp. 53-58, 1989.
[4] P.A. Schweitzer, "Metallic Materials: Physical, Mechanical, and Corrosion Properties", M. Dekker, New York, 2003.

[5] H. Zhang, R. Yang, Y. He, G. Y. Tian, L. Xu, \& $\mathrm{R}$. $\mathrm{Wu}$, "Identification and characterisation of steel corrosion using passive high frequency RFID sensors." Measurement, Vol. 92, pp. 421427, 2016.

[6] K. Niboucha, H. Houali, M. Zergoug, R. Halimi. "Control and characterization of corrosion by CND for the inspection of buried pipelines", Dijon, France, 2006.

[7] S. A. Neild, P. D. McFadden, and M. S. Williams. "A review of time-frequency methods for structural vibration analysis." Engineering Structures, Vol. 25, No .6, pp.713-728, 2003.

[8] P. Flandrin, "Time-frequency", Hermès, Paris, 1993.

[9] L. Cohen, "Time-frequency analysis", Prentice Hall, New York. 1995.

[10] H. M. Ozaktas, N. Erkaya, \& M. A. Kutay, "Effect of fractional Fourier transformation on time-frequency distributions belonging to the Cohen class." IEEE signal processing letter. Vol. 3. No.2, pp.40-41, 1996.

[11] R. Bracewell, "The fourier transform and its applications." New York 5, 1965.

[12] R.W. Revie, H.H. Uhlig, "Corrosion and Corrosion Control: An Introduction to Corrosion Science and Engineering", fourth ed., WileyInterscience, Hoboken, N. J, 2008.

[13] S. Rajakarthihan, S. Kayalvizhy, and K. Gangadevi. "Ultrasonic Investigation on the Effect of Corrosion Inhibitor in Mild Steel." Journal of Pure Applied and Industrial Physics Vol. 2, No. 2, pp 84-141, 2012.

[14] Y. He, G.Y. Tian, M. Pan, D. Chen, H. Zhang, "An investigation into eddy current pulsed thermography for detection of corrosion blister", Corros. Sci. Vol. 78, pp. 1-6, 2014.

[15] A.I. Sunny, G.Y. Tian, J. Zhang, M. Pal, "Low frequency (LF) RFID sensors and selective transient feature extraction for corrosion characterisation", Sens. Actuators A: Phys. Vol. 241, pp. 34-43, 2016.

[16] D. Landolt, " Corrosion and chemical metal surfaces ". Vol. 12. PPUR polytechnic presses, 1997.

[17] W. J. Wang, P. D. McFadden, "Early detection of gear failure by vibration analysis--ii. interpretation of the time-frequency distribution 
using image processing techniques.

" Mechanical Systems and Signal Processing, Vol. 7, No. 3, pp. 205-215, 1993.

[18] R. Latif, M. Laaboubi, E. H. Aassif, and G. Maze. "Dispersion Analysis of Acoustic Circumferential Waves Using Time-Frequency Representations." Vibration and Structural Acoustics Analysis. Springer Netherlands, pp. 183-205, 2011

[19] M. Jabloun. "signal modeling highly nonstationary phase and local amplitude polynomial", National Polytechnic Institute of Grenoble, 2007.

[20] I. Daubechies, "The wavelet transform, timefrequency localization and signal analysis." IEEE transactions on information theory, Vol. 36, No. 5, pp. 961-1005, 1990.

[21] JC. Moore, S. Jevrejev, A. Grinsted, "Application of the cross wavelet transform and wavelet coherence to geophysical time series." Nonlinear processes in geophysics, Vol. 11, No. 5/6, pp. 561-566, 2004.

[22] C. Titry. "Detection by Lamb waves, the hidden corrosion in aircraft aluminum structures ", Thesis University Paris 7, 2006. 